\title{
Effect of Defatted Rice Bran Supplementation on Metabolic Parameters and Inflammatory Status in Overweight/obese Adults with Hypercholesterolemia, a Randomised, Placebo-controlled Intervention
}

Weeraya Saphyakhajorn

Chulalongkorn University Faculty of Allied Health Sciences

Rawiwan Sirirat

Loma Linda University School of Public Health

suwimol sapwarobol ( $\square$ suwimol.sa@chula.ac.th)

Chulalongkorn University Faculty of Allied Health Sciences https://orcid.org/0000-0003-0439-0037

\section{Research Article}

Keywords: defatted rice bran, hypercholesterolemia, overweight, obese

Posted Date: March 7th, 2022

DOI: https://doi.org/10.21203/rs.3.rs-1419572/v1

License: @ (i) This work is licensed under a Creative Commons Attribution 4.0 International License. Read Full License 


\section{Abstract}

Background: Defatted rice bran (DRB), a by-product of rice bran oil production is a rich source of fiber, protein and antioxidant compounds that may pose beneficial effects on metabolic profiles. The current study aimed to investigate the effects of DRB supplementation on anthropometric, blood biochemical indices, dietary intake and inflammatory status in overweight/obese subjects with hypercholesterolemia.

Methods: In a 12-week-randomized placebo-controlled trial, 61 overweight/obese participants with a total cholesterol (TC) level >200 mg/dL were randomly assigned either to $30 \mathrm{~g} / \mathrm{d}$ DRB $(n=30)$ or to $10 \mathrm{~g} / \mathrm{d}$ maltodextrin $(n=31)$.

Results: DRB intervention significantly reduced systolic and diastolic blood pressure by $4.27 \%$ and $4.50 \%$, respectively $(126.20 \pm 13.63$ to $120.60 \pm 13.72 \mathrm{mmHg}$, $p=0.0003$ and $80.87 \pm 7.38$ to $77.17 \pm 9.83 \mathrm{mmHg}, p=0.0035)$. HbA1c also decreased significantly by $3.59 \%(5.89 \pm 0.76$ to $5.66 \pm 0.62 \%, p=0.0001)$ after $\mathrm{DRB}$ supplementation. Total cholesterol, TG and LDL-c levels also decreased insignificantly by $3.12,1.32$ and $1.53 \%$ after DRB supplementation. Insignificant differences of FBG, insulin, HOMA-IR, QUICKI, hs-CRP and homocysteine levels after DRB intervention were also observed. Reduction in caloric and fat intake were reported in DRB groups.

Conclusions: DRB supplementation improved blood pressure and $\mathrm{HbA1C}$ levels. It also lowers blood cholesterol, albeit insignificantly. Caloric and fat intake were also significantly lower after DRB supplementation. Further study is needed to evaluate the mechanisms by which DRB improve these metabolic indices.

Trial registration: Thai Clinical Trial Registration number is TCTR20191020003. Registered 20 October 2019, https://www.clinicaltrials.in.th/.

\section{Background}

Obesity becoming an epidemic where it has been tripled since 1975 [1]. In Thailand, the prevalence of obesity significantly [2]increased from $33.9 \%$ in 2012 to $44.8 \%$ in 2018 [2]. Rice bran is a nutritious by product from rice milling and has been widely used for the animal feeds and rice bran oil production [3]. The process of rice bran oil extraction has long been established. This process not only produces rice bran oil but also a defatted rice bran (DRB) as a main byproduct. A study on physicochemical property of DRB reported the differences in nutrients composition of DRB and full-fat rice bran to some extent. Even though some active ingredients (e.g. oryzanol, phytosterols, polyphenols, tocopherols, and tocotrienols) had been excluded during oil extraction process [4], DRB still holds a substantial amount of nutrients including protein, non-starch polysaccharides, and antioxidant compounds [5, 6]. In addition, the protein digestibility of DRB was higher when compared to that of full fat rice bran [7]. These differences in nutrients composition and protein digestibility might alter the beneficial effects of DRB consumption in comparison to those of full fat rice bran.

In vitro studies showed that rice protein hydrolysate (RPH) lowers blood pressure by inhibiting the angiotensin (ACE) and renin activities [8, 9]. In animal studies, DRB also demonstrated an anti-hypertensive effect by inhibiting ACE activity and increasing nitric oxide (NO) bioavailability. In the study using rats, phytochemicals compounds in DRB posed an anti-inflammatory effect [10,11]. Additionally, DRB demonstrated anti-oxidation in the animal model by reducing plasma malondialdehyde, superoxide production, and suppressed p47phox NADPH oxidase expression in rats fed with a high-carbohydrate and high-fat diet [10]. An anti-diabetic and anti-cholesterolemic effects were also observed in animal studies [12, 13].

Currently, study on the effects of DRB supplementation on those metabolic parameters for humans is limited. With this inadequate information, DRB is currently used as animal feed. Proof of its effect on metabolic indices may provide insight in terms of using DRB as an active ingredient in functional foods. This study therefore aimed to investigate the effects of a DRB supplementation on body weight, lipid profiles, metabolic parameters and inflammatory status in overweight/obese adults with hypercholesterolemia.

\section{Methods}

\subsection{Preparation of DRB}

A mix of brown local Thai rice (Oryza sativa L.) varieties were procured from a local rice mill in the central area of Thailand. A full fat rice bran obtained after a milling process. Thereafter, full fat rice bran underwent heat treatment for stabilization before oil extraction process. In the solvent extraction process, stabilized rice bran is extracted with n-hexane. This procedure provides crude rice bran oil and DRB. The crude rice bran oil contained $41.13 \%$ monounsaturated fatty acids ( $40.6 \%$ oleic acids), $34.24 \%$ polyunsaturated fatty acids $(32.92 \%$ linoleic acids) and $24.63 \%$ saturated fatty acids, $(20.9 \%$ palmitic acids) (Gas Chromatography AOCS 1c-89).Rice bran were heated to 120-130 degree Celsius for 30 seconds via steam and high compression friction. DRB, were powdered, and heated to lower moisture to less than $6 \%$, then passed through a 60 -mesh sieve and stored in air tight containers under hygienic conditions at room temperature, then kept in a dry place until further use. These processes were done at the Thai Ruam Jai Vegetable Oil Co., Ltd. Thailand.

In this clinical trial, DRB was obtained in one batch to maintain homogeneity. For safety purposes, microorganisms (E.coli, S.aureus and coliforms), and other toxic substances (Lead, Cadmium, Arsenic, Alflatoxin) were tested and results showed values within the normal range according to the guidelines of the Thai Food and Drug Administration. Protein content (amino acids), fat, and micro-nutrients composition were determined according to the AOAC standard protocol [14]. Before the clinical trial, 15 grams of DRB was weighed and tightly sealed in an aluminium sachet. Five grams of tapioca-maltodextrin was packed in the same size and type of aluminium sachet to be used as a placebo control. Maltodextrin purchased from Krungthepchemi, Bangkok Thailand. Nutrition composition of DRB (30 grams) and Maltodextrin (10 grams) are shown in Table 1. In this study, 30 grams of DRB provided $90 \mathrm{kcal}, 17.78 \mathrm{grams}$ carbohydrates, 5.55 grams protein, 7.78 grams fiber, and 0 grams fat. Maltodextrin 10 grams provided 40 kcal and 9.5 grams carbohydrates. 
Table 1

The nutritional composition of DRB ( 30 grams) and maltodextrin (10 grams).

\begin{tabular}{|lll|}
\hline Nutrients & $\begin{array}{l}\text { DRB } \\
\text { (30 grams) }\end{array}$ & $\begin{array}{l}\text { Maltodextrin } \\
\text { (10 grams) }\end{array}$ \\
\hline Energy (kcal) & 90 & 40 \\
\hline Carbohydrates (g) & 17.78 & 9.5 \\
\hline Protein (g) & 5.55 & 0 \\
\hline Fat (g) & 0 & 0 \\
\hline Fiber (g) & 7.78 & 0 \\
\hline
\end{tabular}

\subsection{Study design}

Participants were recruited by an advertisement poster in the neighbourhood of Chulalongkorn University, Bangkok, Thailand. A nurse and a registered dietitian screened participants for the inclusion criteria, which included participants who were aged 18 to 60 years old, were overweight or obese with a BMI $\geq 23$ $\mathrm{kg} / \mathrm{m}^{2}$ and fasting total cholesterol (TC) $>200 \mathrm{mg} / \mathrm{dL}$, with no known metabolic-related diseases, no rice bran allergies, and no eating disorders. Participants who smoked, drank alcoholic beverages, had diseases and/or took any medication and dietary supplements related to weight control or could have confounded any study indicators were excluded.

A 12-week, double-blinded, randomized controlled trial was conducted to examine the metabolic properties of DRB in overweight/obese participants with hypercholesterolemia. Sixty-nine participants complied with the inclusion criteria and were randomly allocated (according to www.graphpad.com) to one of the following groups: the intervention (DRB) group $(n=35)$ or the placebo control group $(n=34)$. In the DRB group, five participants withdrew from the study because of lack of follow-up ( $n=3)$, GI disturbance $(n=1)$ and personal reasons $(n=1)$. In the control group, three participants withdrew because of a lack of follow-up ( $n=3)$. In total, 31 participants ( 23 females, 8 males) in the control group and 30 participants ( 21 females, 9 males) in the DRB group completed this study. (Figure 1)

Daily, participants were advised to consume two sachets of DRB (15 grams DRB per sachet) or two sachets of placebo (5 grams maltodextrin per sachet) before a regular meal (breakfast and dinner). During the 12 weeks of intervention, participants were requested to continue their usual diets and maintain their physical activity throughout the study. In addition, they were instructed not to consume any rice bran or rice bran derived products during the study.

After a week-long run-in period, both groups of participants were requested to visit the clinic at the department of nutrition and dietetics, Chulalongkorn University, Bangkok, Thailand, five times: at weeks 0 (baseline), 3, 6, 9 and 12 after intervention to examine the parameters of interest, including blood pressure, anthropometric parameters, and dietary records. Venous blood for the measurement of the parameters of interest including fasting blood glucose (FBG), insulin, HbA1C, fasting blood lipid profiles (TC, TG, HDL-c and LDL-C), and inflammatory cytokines (hs-CRP), and homocysteine levels was drawn at weeks 0,6 and 12. At each clinic visit, the three week's supply of tested foods were distributed, any unused sachets from the previous visit were collected and counted. The participants will be followed up for compliance by randomly phone call two times a week (one weekday and one weekend day).

\subsection{Anthropometric assessment}

Body weight, muscle mass, fat mass and fat free mass were measured using a bioelectrical impedance analyzer (MC-980 MA body composition analyzer, TANITA Corporation, Tokyo, Japan). Participants dress in light attire and bare feet. Eight polar electrodes were positioned, so that electric current was supplied from the electrodes on both feet and hands. Voltage was then measured on the heels of both feet and the near sides of both hands. Waist circumference was measured to the nearest $1.0 \mathrm{~cm}$ using a standard measuring tape at a point right above the iliac crest on the mid-axillary line at minimal respiration. Body mass index (BMI) was calculated as weight/height ${ }^{2}$ (in kilograms per square meter). Blood Pressure was measured using OMRON HEM-8712 blood pressure monitor. Participants were advised to be relaxed and seated for five minutes before the measurement with legs uncrossed and back supported. Blood pressure measurement was duplicated with a 5-minute interval and the average of values was recorded [15]

Visceral adiposity index (VAl) was calculated as described [16] using the following gender-specific equations, when TG is Triglycerides levels expressed in $\mathrm{mmol} / \mathrm{l}$ and $\mathrm{HDL}$ is HDL-Cholesterol levels expressed in $\mathrm{mmol} / \mathrm{l}$ :

$$
\begin{gathered}
\text { Female VAI }=\left(\frac{\text { Waist circumference }(\mathrm{cm})}{36.58+(1.89 \times \mathrm{BMI})}\right) \times\left(\frac{\mathrm{TG}}{0.81}\right) \times\left(\frac{1.52}{\mathrm{HDL}}\right) \\
\text { Male VAI }=\left(\frac{\text { Waist circumference }(\mathrm{cm})}{39.68+(1.88 \times \mathrm{BMI})}\right) \times\left(\frac{\mathrm{TG}}{1.03}\right) \times\left(\frac{1.31}{\mathrm{HDL}}\right)
\end{gathered}
$$

Relative fat mass (RFM) was calculated by using the following equation:

$$
\mathrm{RFM}=64-\left(20 \times \frac{\text { height }(\mathrm{m})}{\text { waist }(\mathrm{m})}\right)+(12 \times \text { gender })
$$

When height and waist circumference are expressed in meters. Gender $=0$ for male and 1 for female [17].

\subsection{Blood biochemical assessment}


At each clinic visit, approximately $15 \mathrm{ml}$. blood samples were taken from a vein puncture by medical technologists and nurses after an overnight fast of 10 to 12 hours. After collection, blood samples were separated into four tubes. For fasting glucose concentration determination, blood samples were kept in sodiumfluoride tubes. For \% HbA1c and homocysteine determination, samples were kept in EDTA tubes. In addition, for fasting lipid, insulin and hs-CRP determination, blood samples were kept in two tubes of clot activator.

Blood glucose was examined by the hexokinase method using a clinical chemistry analyser (Beckman Coulter AU480, USA), whereas TC, LDL-c, HDL-c and TG were examined using the enzymatic method (Beckman Coulter, USA). Serum insulin levels were analysed by the chemiluminescence immunoassay method (CLIA) [18]. Blood samples were immediately centrifuged (3,000 rpm) for $10 \mathrm{~min}$ at $4^{\circ} \mathrm{C}$ and examined on the same day of blood collection. For serum hs-CRP and homocysteine analysis, blood samples were immediately centrifuged $(3,000 \mathrm{rpm})$ for $10 \mathrm{~min}$ at $4^{\circ} \mathrm{C}$, and the specimens were kept at $-80^{\circ} \mathrm{C}$ for further analysis. Serum hs-CRP was measured by turbidmetric immunoinhibition assay (Beckman Coulter, USA). Serum homocysteine was analysed by the chemiluminescense immunoassay method (Abbott Diagnostics).

All metabolic outcomes were examined at a Health Sciences service unit, Faculty of Allied Health Sciences, Chulalongkorn University. Additionally, the homeostatic model assessment of insulin resistance (HOMA-IR) was calculated as a fasting serum insulin $(\mu \mathrm{IU} / \mathrm{mL}) \times \mathrm{fasting} \mathrm{plasma} \mathrm{glucose}(\mathrm{mg} / \mathrm{dL}) / 405$. A quantitative insulin sensitivity check index (QUICKI) was calculated as a log transform of the insulin glucose product. QUICKI $=1 /[$ log(fasting insulin) $+\log ($ fasting glucose) $][19,20]$.

\subsection{Dietary intake assessment}

A weekly (two weekdays and one weekend) diet record was collected and examined for average intakes throughout the 12 weeks of the intervention period. Energy and macronutrient intake was calculated by using food composition database in INMUCAL Nutrients software version 3 (developed by the Institute of Nutrition, Mahidol University, Thailand), which is based on Thai food composition and recipes [21]. The average daily intake of energy, carbohydrates, protein, fat, and dietary fiber of the DRB and placebo groups were presented as an average of energy and nutrients recorded in the week prior to the study (which represents the baseline data), as well as during the study.

\subsection{Gastrointestinal symptoms assessment}

Participants were instructed to record their gastrointestinal symptoms including flatulence, borborygmi, nausea, vomiting, stomach pain, and passing flatus by means of a gastrointestinal symptom questionnaire. Participants rated the intensity of symptoms from 0 (none), 1 (mild), 2 (moderate), to 3 (severe). Total score was calculated for the intensity of all symptoms. Participants also evaluated their stool form by using the Bristol Stool Scale with a picture and description for each type of stool form [22].

\subsection{Statistical analysis}

The sample size was calculated based on the difference of the serum total cholesterol between the groups from the previous study of Hongu et al. [23], and the power and alpha levels set at $80 \%$ and at 0.05 , respectively. A sample size of 29 participants (in each group) was considered adequate. Statistical analyses were conducted using SPSS software for Windows (version 22.0; SPSS, Inc., Chicago, IL). The normal distribution of the values was checked by a Kolmogorov-Smirnov test. Continuous variables were presented as the means and standard deviations, while categorical data were presented as numbers and percentages. The categorical variables were compared with a Chi-square test. An independent t-test was used to compare continuous variables at the beginning of the study and mean changes of these variables during the intervention between the two groups. To analyse group changes at the baseline and follow-up weeks, a repeat-measured ANOVA was used. Tukey's multiple comparison test was used to compare the groups when ANOVA test results were significant. All statistical analyses were 2-sided and evaluated at $p=0.05$.

\section{Results}

Defatted rice bran used in this study were powdered, and heated to lower moisture to less than $6 \%$, then passed through a 60 -mesh sieve and stored in air-tight containers under hygienic conditions at room temperature, then kept in a dry place until given to participants. At baseline, there was no significant difference in anthropometric, blood biochemical and dietary intake parameters between the placebo $(n=31,23$ females, 8 males) and DRB ( $n=30,21$ females, 9 males) groups. However, HDL-c at the baseline was significantly higher in DRB participants $(57.7 \pm 13.21 \mathrm{mg} / \mathrm{dL})$ than in the placebo group $(51.35 \pm 10.21 \mathrm{mg} / \mathrm{dL}) p=$ 0.0397 (Table 2). 
Table 2

Baseline characteristics of the placebo $(n=31)$ and DRB $(n=30)$ groups.

\begin{tabular}{|c|c|c|}
\hline Parameters & Placebo $(n=31)$ & DRB $(n=30)$ \\
\hline \multicolumn{3}{|l|}{ Anthropometrics parameters } \\
\hline Age (years) & $31.71 \pm 12.27$ & $36.87 \pm 12.30$ \\
\hline \multicolumn{3}{|l|}{ Gender } \\
\hline Female & $23(74.2 \%)$ & $21(70.0 \%)$ \\
\hline Male & $8(25.8 \%)$ & $9(30.0 \%)$ \\
\hline Height (cm) & $163.48 \pm 9.16$ & $162.08 \pm 8.26$ \\
\hline Body weight (kg) & $75.38 \pm 15.56$ & $77.76 \pm 16.75$ \\
\hline $\mathrm{BMI}\left(\mathrm{kg} / \mathrm{m}^{2}\right)$ & $28.10 \pm 4.50$ & $29.45 \pm 4.57$ \\
\hline Waist circumference $(\mathrm{cm})$ & $93.60 \pm 11.03$ & $95.09 \pm 10.86$ \\
\hline Fat mass $(\mathrm{kg})$ & $27.68 \pm 10.26$ & $30.21 \pm 10.20$ \\
\hline Fat free mass (kg) & $47.74 \pm 10.82$ & $47.48 \pm 10.43$ \\
\hline Muscle mass $(\mathrm{kg})$ & $45.02 \pm 10.38$ & $44.52 \pm 10.48$ \\
\hline Relative fat mass & $37.60 \pm 6.63$ & $38.30 \pm 5.58$ \\
\hline Visceral adiposity index & $2.07 \pm 1.00$ & $1.64 \pm 0.96$ \\
\hline $\mathrm{SBP}(\mathrm{mmHg})$ & $122.13 \pm 15.05$ & $126.20 \pm 13.63$ \\
\hline $\mathrm{DBP}(\mathrm{mmHg})$ & $78.45 \pm 10.32$ & $80.87 \pm 7.38$ \\
\hline \multicolumn{3}{|c|}{ Blood biochemical parameters } \\
\hline $\mathrm{FBG}(\mathrm{mg} / \mathrm{dL})$ & $99.13 \pm 27.95$ & $94.93 \pm 22.79$ \\
\hline $\mathrm{HbA1c}(\%)$ & $5.89 \pm 0.67$ & $5.89 \pm 0.76$ \\
\hline Serum Insulin (ulU/mL) & $9.16 \pm 4.28$ & $8.50 \pm 4.37$ \\
\hline HOMA-IR & $2.10 \pm 1.04$ & $2.14 \pm 1.50$ \\
\hline QUICKI & $0.35 \pm 0.03$ & $0.35 \pm 0.03$ \\
\hline $\mathrm{TC}(\mathrm{mg} / \mathrm{dL})$ & $236.32 \pm 30.44$ & $242.00 \pm 46.45$ \\
\hline $\mathrm{TG}(\mathrm{mg} / \mathrm{dL})$ & $131.27 \pm 58.99$ & $121.52 \pm 64.94$ \\
\hline LDL-c (mg/dL) & $158.94 \pm 33.57$ & $165.40 \pm 37.69$ \\
\hline HDL-c (mg/dL) & $51.35 \pm 10.21$ & $57.7 \pm 13.21^{\star}$ \\
\hline LDL:HDL ratio & $3.19 \pm 0.80$ & $3.06 \pm 0.87$ \\
\hline $\mathrm{hs}-\mathrm{CRP}(\mathrm{mg} / \mathrm{L})$ & $2.74 \pm 1.91$ & $1.88 \pm 1.59$ \\
\hline Homocysteine $(\mu \mathrm{mol} / \mathrm{L})$ & $10.69 \pm 3.07$ & $11.37 \pm 3.29$ \\
\hline \multicolumn{3}{|l|}{ Dietary intake } \\
\hline Energy (kcal/day) & $1,689.29 \pm 428.64$ & $1,770.4 \pm 257.16$ \\
\hline Carbohydrate (g/day) & $212.29 \pm 57.58$ & $225.37 \pm 45.77$ \\
\hline Protein (g/day) & $74.09 \pm 31.16$ & $71.94 \pm 15.24$ \\
\hline Fat (g/day) & $60.81 \pm 20.72$ & $64.80 \pm 16.39$ \\
\hline \multicolumn{3}{|l|}{ Energy distribution } \\
\hline Carbohydrate (\%) & $51.35 \pm 7.03$ & $51.35 \pm 7.53$ \\
\hline Protein (\%) & $17.10 \pm 4.58$ & $16.37 \pm 2.86$ \\
\hline Fat (\%) & $31.55 \pm 6.04$ & $32.28 \pm 6.37$ \\
\hline \multicolumn{3}{|c|}{ Gastrointestinal symptoms parameters } \\
\hline Flatulence & $0.4 \pm 0.82$ & $0.41 \pm 0.68$ \\
\hline Borborygmi & $0.63 \pm 0.72$ & $0.59 \pm 0.82$ \\
\hline
\end{tabular}

Page 5/13 


\begin{tabular}{|lll|}
\hline Parameters & Placebo $(\mathbf{n = 3 1})$ & DRB $(\mathbf{n = 3 0})$ \\
\hline Nausea & $0.07 \pm 0.25$ & $0.10 \pm 0.56$ \\
\hline Vomiting & $0.00 \pm 0.00$ & $0.07 \pm 0.37$ \\
\hline Stomach pain & $0.27 \pm 0.69$ & $0.17 \pm 0.47$ \\
\hline Passing flatus & $0.73 \pm 0.91$ & $0.66 \pm 0.81$ \\
\hline Bristol stool form & $4.37 \pm 1.38$ & $4.17 \pm 1.23$ \\
\hline
\end{tabular}

All values are expressed as the mean \pm SD. Significant differences between categorical variables of the two study groups were determined by the Chi-square test. Significant differences between continuous variables of the two study groups were determined by independent $t$-tests. ${ }^{*} P$-value $\leq 0.05$ is considered as a statistically significant. SBP=Systolic blood pressure, DBP=Diastolic blood pressure, BMI=Body mass index, FBG=Fasting blood glucose, HOMA-IR=The homeostatic model assessment of insulin resistance, QUICKI=The quantitative insulin-sensitivity check index, TC=Total cholesterol, TG=Triglycerides, LDLc=Low Density Lipoprotein, HDL-c=High density lipoprotein.

\subsection{Anthropometric parameters}

The study did not show any significant differences in body weight between the DRB and placebo group after 12 weeks of intervention: ( $77.76 \pm 16.75 \mathrm{~kg}$ to $77.99 \pm 16.51 \mathrm{~kg}$ and $75.38 \pm 15.56 \mathrm{~kg}$ to $75.28 \pm 15.29 \mathrm{~kg}$, respectively). Likewise, no significant alterations on the remaining body composition parameters between groups were revealed (Table 3). However, systolic blood pressure was significantly decreased by $4.27 \%$ after 12 weeks of DRB supplementation $(126.20 \pm 13.63$ to $120.60 \pm 13.72 \mathrm{mmHg}, p=0.0003)$. Moreover, the diastolic blood pressure of participants supplemented with DRB significantly decreased $4.50 \%$ after intervention compared to that of the baseline $(80.87 \pm 7.38 \mathrm{vs}$. $77.17 \pm 9.83 \mathrm{mmHg}, p=0.0035)$, while there were no significant changes in blood pressure in the placebo group.

\subsection{Blood biochemical parameters}

Total Cholesterol, TG and LDL-c levels decreased insignificantly by $3.12,1.32$ and $1.53 \%$ after DRB supplementation $(246.40 \pm 45.22$ to $238.27 \pm 47.31 \mathrm{mg} / \mathrm{dL}$, $121.52 \pm 64.94$ to $112.24 \pm 54.46 \mathrm{mg} / \mathrm{dL}$ and $168.73 \pm 37.59$ to $166.27 \pm 41.54 \mathrm{mg} / \mathrm{dL}$, respectively). The LDL:HDL ratio also improved insignificantly from $3.06 \pm 0.87$ (at baseline) to $3.02 \pm 0.86$ after 12 weeks of DRB intervention. At week 12, there were no significant differences in FBG, insulin, HOMA-IR and QUICKI between the DRB and placebo groups $(96.57 \pm 22.40 \mathrm{vs}$. $101.19 \pm 31.79 \mathrm{mg} / \mathrm{dL}, 8.38 \pm 3.88 \mathrm{vs} .8 .99 \pm 5.04 \mathrm{ulU} / \mathrm{mL}, 2.15 \pm 1.53 \mathrm{vs}$. $2.10 \pm 1.29$ and $0.35 \pm 0.03 \mathrm{vs}$. $0.35 \pm 0.04$, respectively). However, HbA1C level significantly decreased by $-3.59 \%(5.89 \pm 0.76$ to $5.66 \pm 0.62 \%, p=0.0001)$ in participants supplemented with DRB. In addition, the effect of DRB on lowering HbA1C levels was observed as early as week 6 (Table 3). Additionally, there was no significant difference of the hs-CRP concentration between the control and DRB groups after 12 weeks of intervention ( $2.79 \pm 2.35 \mathrm{vs}$. $2.09 \pm 1.94 \mathrm{mg} / \mathrm{L}$, respectively). In addition, hs-CRP concentrations of participants in the DRB group at week 12 was insignificantly different when compared to that of the baseline $(1.88 \pm 1.59$ to $2.09 \pm 1.94, p=$ 0.0970). Similarly, the concentration of homocysteine was not significantly different when compared between the control and DRB groups at baseline (10.69 \pm $3.07 \mu \mathrm{mol} / \mathrm{L}$ vs. $11.37 \pm 3.29 \mu \mathrm{mol} / \mathrm{L})$ and after 12 weeks of intervention $(11.06 \pm 2.46 \mu \mathrm{mol} / \mathrm{L}$ vs. $10.98 \pm 3.20 \mu \mathrm{mol} / \mathrm{L})($ Table 3$)$. 
Table 3

Comparison of Anthropometrics, blood biochemical and dietary intake parameters of the placebo $(n=31)$ and DRB $(n=30)$ groups.

\begin{tabular}{|c|c|c|c|c|c|c|c|}
\hline \multirow[t]{2}{*}{ Parameters } & \multicolumn{3}{|l|}{ Placebo $(n=31)$} & \multirow[t]{2}{*}{ Mean change } & \multicolumn{3}{|l|}{ DRB $(n=30)$} \\
\hline & Baseline & Week 6 & Week 12 & & Baseline & Week 6 & Week 12 \\
\hline \multicolumn{8}{|c|}{ Anthropometrics parameters } \\
\hline $\begin{array}{l}\text { Body weight } \\
\text { (kg) }\end{array}$ & $75.38 \pm 15.56$ & $75.3 \pm 15.63$ & $75.28 \pm 15.29$ & $-0.10 \pm 1.80$ & $77.76 \pm 16.75$ & $78.00 \pm 16.60$ & $77.99 \pm 16.51$ \\
\hline BMI $\left(\mathrm{kg} / \mathrm{m}^{2}\right)$ & $28.10 \pm 4.50$ & $28.12 \pm 4.56$ & $28.13 \pm 4.56$ & $0.03 \pm 0.71$ & $29.45 \pm 4.57$ & $29.52 \pm 4.64$ & $29.55 \pm 4.66$ \\
\hline $\begin{array}{l}\text { Waist } \\
\text { circumference } \\
\text { (cm) }\end{array}$ & $93.60 \pm 11.03$ & $93.31 \pm 11.24$ & $93.44 \pm 11.27$ & $-0.16 \pm 1.19$ & $95.09 \pm 10.86$ & $95.40 \pm 11.06$ & $95.28 \pm 10.81$ \\
\hline Fat mass $(\mathrm{kg})$ & $27.68 \pm 10.26$ & $27.59 \pm 10.54$ & $27.43 \pm 10.03$ & $-0.25 \pm 1.68$ & $30.21 \pm 10.20$ & $30.26 \pm 10.52$ & $30.46 \pm 10.26$ \\
\hline $\begin{array}{l}\text { Fat free mass } \\
(\mathrm{kg})\end{array}$ & $47.74 \pm 10.82$ & $47.82 \pm 10.70$ & $47.69 \pm 10.99$ & $-0.05 \pm 1.16$ & $47.48 \pm 10.43$ & $47.48 \pm 10.25$ & $47.60 \pm 10.41$ \\
\hline $\begin{array}{l}\text { Muscle mass } \\
(\mathrm{kg})\end{array}$ & $45.02 \pm 10.38$ & $45.10 \pm 10.25$ & $45.03 \pm 10.62$ & $0.02 \pm 1.09$ & $44.52 \pm 10.48$ & $44.90 \pm 9.94$ & $44.93 \pm 10.02$ \\
\hline $\begin{array}{l}\text { Relative fat } \\
\text { mass }\end{array}$ & $37.60 \pm 6.63$ & $37.47 \pm 6.66$ & $37.52 \pm 6.62$ & $-0.08 \pm 0.49$ & $38.30 \pm 5.58$ & $38.39 \pm 5.69$ & $38.37 \pm 5.68$ \\
\hline $\begin{array}{l}\text { Visceral } \\
\text { adiposity } \\
\text { index }\end{array}$ & $2.07 \pm 1.00$ & $2.07 \pm 0.96$ & $2.01 \pm 0.99$ & $-0.04 \pm 0.69$ & $1.64 \pm 0.96$ & $1.50 \pm 0.67$ & $1.54 \pm 0.93$ \\
\hline $\mathrm{SBP}(\mathrm{mmHg})$ & $122.13 \pm 15.05$ & $121.32 \pm 14.60$ & $123.52 \pm 13.93$ & $1.39 \pm 9.67$ & $126.20 \pm 13.63^{a}$ & $123.33 \pm 13.07^{a, b}$ & $120.60 \pm 13.72^{b}$ \\
\hline $\mathrm{DBP}(\mathrm{mmHg})$ & $78.45 \pm 10.32$ & $80.00 \pm 8.19$ & $79.19 \pm 8.81$ & $0.74 \pm 7.13$ & $80.87 \pm 7.38^{a}$ & $77.40 \pm 10.89^{b}$ & $77.17 \pm 9.83^{c}$ \\
\hline \multicolumn{8}{|c|}{ Blood biochemical parameters } \\
\hline $\mathrm{FBG}(\mathrm{mg} / \mathrm{dL})$ & $99.13 \pm 27.95$ & $100.35 \pm 30.88$ & $101.19 \pm 31.79$ & $2.06 \pm 9.27$ & $94.93 \pm 22.79$ & $96.60 \pm 21.98$ & $96.57 \pm 22.40$ \\
\hline HbA1c (\%) & $5.89 \pm 0.67^{a}$ & $5.81 \pm 0.79^{a, b}$ & $5.78 \pm 0.69^{b}$ & $-0.11 \pm 0.18$ & $5.89 \pm 0.76^{a}$ & $5.77 \pm 0.70^{b}$ & $5.66 \pm 0.62^{c}$ \\
\hline $\begin{array}{l}\text { Insulin } \\
\text { (ulU/mL) }\end{array}$ & $9.16 \pm 4.28$ & $8.86 \pm 4.59$ & $8.99 \pm 5.04$ & $-0.35 \pm 2.87$ & $8.50 \pm 4.37$ & $8.25 \pm 3.26$ & $8.38 \pm 3.88$ \\
\hline HOMA-IR & $2.10 \pm 1.04$ & $2.06 \pm 1.08$ & $2.10 \pm 1.29$ & $0.07 \pm 0.77$ & $2.14 \pm 1.50$ & $2.13 \pm 1.46$ & $2.15 \pm 1.53$ \\
\hline QUICKI & $0.35 \pm 0.03$ & $0.35 \pm 0.04$ & $0.35 \pm 0.04$ & $0.00 \pm 0.02$ & $0.35 \pm 0.03$ & $0.35 \pm 0.04$ & $0.35 \pm 0.03$ \\
\hline $\mathrm{TC}(\mathrm{mg} / \mathrm{dL})$ & $236.32 \pm 30.44$ & $230.94 \pm 34.40$ & $238.03 \pm 36.59$ & $1.71 \pm 20.03$ & $246.40 \pm 45.22$ & $242.00 \pm 46.45$ & $238.27 \pm 47.31$ \\
\hline TG $(\mathrm{mg} / \mathrm{dL})$ & $131.27 \pm 58.99$ & $133.4 \pm 59.42$ & $130.10 \pm 53.52$ & $2.00 \pm 43.71$ & $121.52 \pm 64.94$ & $112 \pm 46.87$ & $112.24 \pm 54.46$ \\
\hline $\mathrm{LDL}(\mathrm{mg} / \mathrm{dL})$ & $158.94 \pm 33.57^{\mathrm{a}}$ & $164.58 \pm 36.83^{a, b}$ & $169.58 \pm 35.11^{b}$ & $10.65 \pm 22.55$ & $168.73 \pm 37.59$ & $165.40 \pm 37.69$ & $166.27 \pm 41.54$ \\
\hline $\mathrm{HDL}(\mathrm{mg} / \mathrm{dL})$ & $51.35 \pm 10.21$ & $52.35 \pm 11.10$ & $53.23 \pm 11.69$ & $1.87 \pm 6.32$ & $57.7 \pm 13.21$ & $56.97 \pm 14.42$ & $57.63 \pm 14.73$ \\
\hline LDL:HDL ratio & $3.19 \pm 0.80$ & $3.25 \pm 0.84$ & $3.32 \pm 0.93$ & $0.14 \pm 0.38$ & $3.06 \pm 0.87$ & $3.04 \pm 0.87$ & $3.02 \pm 0.86$ \\
\hline $\begin{array}{l}\text { hs-CRP } \\
\text { (mg/L) }\end{array}$ & $2.74 \pm 1.91$ & $3.11 \pm 3.30$ & $2.79 \pm 2.35$ & $-0.55 \pm 3.21$ & $1.88 \pm 1.59$ & $2.28 \pm 1.83$ & $2.09 \pm 1.94$ \\
\hline $\begin{array}{l}\text { Homocysteine } \\
(\mu \mathrm{mol} / \mathrm{L})\end{array}$ & $10.69 \pm 3.07$ & $10.37 \pm 2.87$ & $11.06 \pm 2.46$ & $0.36 \pm 2.99$ & $11.37 \pm 3.29$ & $11.10 \pm 3.12$ & $10.98 \pm 3.20$ \\
\hline \multicolumn{8}{|l|}{$\begin{array}{l}\text { Dietary intake } \\
\text { parameters }\end{array}$} \\
\hline $\begin{array}{l}\text { Energy } \\
\text { (kcal/day) }\end{array}$ & $1,689.29 \pm 428.64$ & $1,715.08 \pm 460.73$ & $1,777.30 \pm 444.95$ & $88.01 \pm 949.19$ & $1,770.40 \pm 257.16^{a}$ & $1,723.56 \pm 374.34^{a, b}$ & $1,646.16 \pm 339.8$ \\
\hline $\begin{array}{l}\text { Carbohydrate } \\
\text { (g/day) }\end{array}$ & $212.29 \pm 57.58$ & $230.65 \pm 77.81$ & $233.93 \pm 178.47$ & $21.64 \pm 184.05$ & $225.37 \pm 45.77$ & $226.04 \pm 42.55$ & $216.55 \pm 51.27$ \\
\hline $\begin{array}{l}\text { Protein } \\
\text { (g/day) }\end{array}$ & $74.09 \pm 31.16$ & $69.65 \pm 19.11$ & $72.00 \pm 32.94$ & $-2.09 \pm 23.98$ & $71.94 \pm 15.24$ & $72.54 \pm 17.62$ & $71.45 \pm 14.28$ \\
\hline Fat (g/day) & $60.81 \pm 20.72$ & $56.72 \pm 18.77$ & $60.86 \pm 26.05$ & $0.05 \pm 25.33$ & $64.80 \pm 16.39^{a}$ & $58.84 \pm 22.20^{a, b}$ & $59.62 \pm 21.88^{b}$ \\
\hline \multicolumn{8}{|l|}{$\begin{array}{l}\text { Energy } \\
\text { distribution }\end{array}$} \\
\hline $\begin{array}{l}\text { Carbohydrate } \\
(\%)\end{array}$ & $51.35 \pm 7.03$ & $53.12 \pm 7.22$ & $55.22 \pm 28.18$ & $3.87 \pm 29.82$ & $51.35 \pm 7.53$ & $51.63 \pm 6.31$ & $51.08 \pm 5.47$ \\
\hline
\end{tabular}




\begin{tabular}{|c|c|c|c|c|c|c|c|}
\hline \multirow[t]{2}{*}{ Parameters } & \multicolumn{3}{|c|}{ Placebo $(n=31)$} & \multirow[t]{2}{*}{ Mean change } & \multicolumn{3}{|l|}{ DRB $(n=30)$} \\
\hline & Baseline & Week 6 & Week 12 & & Baseline & Week 6 & Week 12 \\
\hline Protein (\%) & $17.10 \pm 4.58$ & $16.77 \pm 3.06$ & $17.99 \pm 5.07$ & $0.88 \pm 4.90$ & $16.37 \pm 2.86$ & $16.55 \pm 2.47$ & $17.34 \pm 2.75$ \\
\hline Fat (\%) & $31.55 \pm 6.04$ & $30.11 \pm 6.06$ & $33.46 \pm 8.33$ & $1.92 \pm 9.24$ & $32.28 \pm 6.37$ & $31.82 \pm 6.26$ & $31.58 \pm 6.06$ \\
\hline $\begin{array}{l}\text { Dietary fiber } \\
\text { (g/day) }\end{array}$ & $6.98 \pm 3.28$ & $6.25 \pm 2.88$ & $7.47 \pm 5.91$ & $0.49 \pm 5.57$ & $9.48 \pm 5.35^{a}$ & $15.40 \pm 2.99^{b}$ & $15.38 \pm 3.33^{c x}$ \\
\hline
\end{tabular}

All values are expressed as the mean \pm SD. Significant difference at each follow-up time within a group were determined by repeated measures ANOVA. Significant difference between two study groups were determined by independent t-tests. Different letters on the same row refers to significant different at each follow-up week. * refers to significant different between study group at week 12 . P-value $\leq 0.05$ is considered statistically significant for all tests. $\mathrm{BMI}=$ Body mass index, SBP=Systolic blood pressure, DBP=Diastolic blood pressure, FBG=fasting blood glucose, HOMA-IR=the homeostatic model assessment of insulin resistance, QUICKI=the quantitative insulin sensitivity check index, TC=total cholesterol, TG=triglycerides, LDL=low-density lipoprotein, HDL=high-density lipoprotein, hs-CRP = high sensitivity C-reactive protein.

\subsection{Dietary intake parameters}

The average energy intake in the DRB group decreased significantly from the baseline to the end of the study $(1,770.4 \pm 257.16 \mathrm{vs}$. $1,646.16 \pm 339.87 \mathrm{kcal} / \mathrm{d}$., $p=0.0120)$. In addition, participants in the DRB group also reported less consumption of carbohydrates and fat $(225.37 \pm 45.77 \mathrm{~g} / \mathrm{day}$ and $64.80 \pm 16.39 \mathrm{~g} / \mathrm{day}$ at baseline to $216.55 \pm 51.27 \mathrm{~g} /$ day and $59.62 \pm 21.88 \mathrm{~g} /$ day, respectively). Additionally, supplementation of DRB significantly increased the mean dietary fiber intake from $9.48 \pm 5.35 \mathrm{~g} /$ day at the baseline to $15.38 \pm 3.33 \mathrm{~g} /$ day after 12 weeks of intervention $(p<0.0001)$ (Table 3).

\subsection{Gastrointestinal symptoms parameters}

The result showed that $96.55 \%$ of participants in the DRB group reported no gastrointestinal symptoms after supplementation, while $3.45 \%$ reported mild gastrointestinal symptoms including flatulence, borborygmi, nausea, stomach pain, and passing flatus (Figure 2). Participants in DRB group reported an improvement in a healthy stool form (type 4 stool form) from $34.48 \%$ at baseline to $48.28 \%$, while there was no change in type 4 stool form in control group (35.48-32.26\%) (Figure 3).

\section{Discussion}

The present study reported that 30 grams of DRB supplementation daily for 12 weeks does not significantly alter body weight and others body composition indices. In accordance with a systematic review of randomized controlled trials which reported that fiber consumption had insignificant effect on energy intake and body weight [24]. Even though, it was reported that soluble fiber reduce appetite and increase satiety, limited amount of soluble fiber (6.16\% (w/w)) contained in DRB may be an explanation for these null outcomes.

Daily DRB supplementation effectively reduced both systolic and diastolic blood pressure in overweight and obese adults with hypercholesterolemia. The in vitro study showed that rice bran peptide hydrolysate (RBPH) of molecular sized $>50$ and 10-50 kDa could inhibit angiotension- 1 converting enzyme (ACE) by $78 \%$ and $55 \%$, respectively [25]. The plausible mechanism of rice bran protein on blood pressure includes ACE inhibitory activity, the enhancement of the eNOS pathway, an increase in NO bioavailability and the attenuation of ROS formation through the inhibition of the NADPH oxidase system [10, 11]. The 3 peptides, Leu-Arg-Ala, contained in rice bran has been demonstrated to induce vasorelaxation mediated the NO pathway in the endothelium of blood vessels [26].

This study demonstrated that DRB supplementation reduced HbA1c concentration by $3.59 \%$. There are various possible mechanisms for this improvement including enhanced secretion of glucose-dependent insulintropic polypeptide (GIP) [27], reduced appetite and food intake [28] and inhibited GLUT 4 transporters [29]. In addition, it was well established that insoluble fiber may increase faecal bulk and decrease intestinal transit time, thus resulting in a decrease absorption of glucose and other simple carbohydrates and an 8\% improvement of insulin sensitivity [30].

In this study, DRB supplementation had an insignificant effect on FBG, serum insulin, HOMA-IR, and QUICKI. Even though previous studies have demonstrated a reduction in FBG after DRB supplementation, the majority of them were conducted with Type I or II diabetes mellitus patients [31, 32]. The normoglycaemic status at baseline and tightly control glucose homeostasis in healthy young adults in this study may be partly responsible for these null effects.

Even though, cholesterol-lowering properties was reported in a full fat-rice bran supplementation study. This study observed insignificantly reduction on TC, TG and LDL-c concentrations by $3.12 \pm 9.47 \%, 1.32 \pm 24.86 \%$ and $1.53 \pm 10.90 \%$, respectively after DRB supplementation. This null effect might be because of limited amount of unsaponifiable compounds ( $\gamma$-oryzanol, $\beta$-sitosterol and tocotrienols) contained in DRB. These unsaponifiable compounds had been reported to responsible for the cholesterol lowering properties [32]. Since these compounds have similar structures to that of cholesterol, they may compete with cholesterol absorption in the small intestine [33]. Furthermore, $\beta$ - and $\gamma$-tocotrienols can inhibit 3-hydroxy-3-methyl-glutaryl-coenzyme A (HMG-CoA) reductase, thus reducing endogenous cholesterol synthesis [34]. During the oil-extraction, unsaponifiable compounds were excluded to some extent. With the limited amount of these compounds, DRB may not effectively improve blood lipid profiles. This study therefore observed only a trend of DRB supplementation on cholesterol lowering effect.

A mean reduction in daily energy intake $(120 \mathrm{kcal})$ and dietary fat were observed whereas carbohydrate and protein consumption remained constant. This effect might be a consequence of an increase in dietary fiber consumption of 7.78 grams ( 7.27 grams insoluble and 0.51 grams soluble). It has been proved that insoluble fiber can reduces appetite and increase fat satiety which consequently decreases caloric and fat intake [28]. 
This study demonstrated null effect of DRB on hs-CRP, an anti-inflammatory cytokines and homocysteine. Previous studies showed that phytochemicals and unsaponifiable compounds exhibit a potent free radical scavenging activity $[35,36]$. It was also reported that rice bran polysaccharide increased enzyme antioxidant system in mice while decreased the MDA content [37]. With limited amounts of these beneficial compounds and its components after oil extraction process of rice bran, DRB posed insignificant effect on hs-CRP concentrations. In addition, the amounts of vitamin B6 in 30 grams of DRB may have been inadequate to significantly lower levels of homocysteine. Additionally, the amount of vitamin B6 in the 30 grams DRB may not be the exclusive solution for improving homocysteine levels.

The present study reported that DRB supplementation does not caused gastrointestinal disturbance. However, an improvement in a healthy stool form was reported. As mentioned previously, DRB contains mainly insoluble fiber which produces the stool bulk effect and reduces intestinal transit time [38-40]. Additionally, another study by Tomlin and Read showed that rice bran increased stool mass and stool frequency after supplementing for 10 days. They also suggested that the stool bulking effect of rice bran is caused by a high content of insoluble fiber [41].

The present study used a randomised controlled trial to minimise bias. It also provided information about the effects of DRB supplementation on anthropometrics, blood biochemical parameters and dietary intake in overweight/obese adults with hypercholesterolemia. The results of this study will benefit the food manufacturing sector by providing information on using DRB as an active ingredient in functional foods. However, there were some limitations. First, it did not measure the physical activity, a significant confounding factor during the intervention period. Second, spontaneous improvement of placebo in a randomized design without cross-over was another major limitation in this study. Third, it recruited overweight/obese adults with hypercholesterolemia otherwise healthy participants; therefore, this result cannot be generalised to other population, and so it cannot apply to any diabetes mellitus patients. Further studies related to the mechanism of DRB on metabolic effects are necessary to describe a clear picture of DRB and its potential use in industrial sector.

\section{Conclusions}

DRB could be incorporated as a functional food ingredient to significantly improve blood pressure. It improves HbA1C levels and lower calorie and fat intake. On the other hand, DRB has no significant effect on lowering blood cholesterol levels. Further study is needed to evaluate the mechanisms of DRB supplementation on these beneficial metabolic changes.

\section{Abbreviations}

\section{ACE}

angiotensin-converting enzyme

BMI

body mass index

CLIA

chemiluminescence immunoassay method

DRB

defatted rice bran

FBG

fasting blood glucose

GIP

glucose-dependent insulintropic polypeptide

HMG-CoA

3-hydroxy-3-methyl-glutaryl-coenzyme A

HOMA-IR

homeostatic model assessment of insulin resistance

NO

nitric oxide

QUICKI

quantitative insulin sensitivity check index

RFM

relative fat mass

$\mathrm{RPH}$

rice protein hydrolysate

$\mathrm{TC}$

fasting total cholesterol

TG

triglycerides

VAI

visceral adiposity index

\section{Declarations}




\section{Ethics approval and consent to participate}

All procedures involving human participants were approved by the Ethical Review Committee for Human Research, Faculty of Public Health, Mahidol University, Bangkok, Thailand (MUPH 2017-220). All methods were performed in accordance with the relevant guidelines and regulations. Participants were informed about the details of the study, procedures, and adverse effects of the study product. After the randomization, participants were referred by identification number. Written informed consent was obtained from all participants prior to enrolment in the study. The anonymity of the participants was preserved. Trial registration: TCTR20191020003. Registered 20 October 2019, https://www.clinicaltrials.in.th/.

\section{Consent for publication}

Not applicable

\section{Availability of data and materials}

The datasets used and/or analysed during the current study are available from the corresponding author on reasonable request.

\section{Competing interests}

The authors declare that they have no competing interests.

\section{Funding}

This work was financially supported by Thai Ruam Jai Vegetable Oil Co., Ltd. Suwimol Sapwarobol received a travelling expense from Chulalongkorn University Office of International Affairs Scholarships for short-term research visit in United States. Weeraya Saphyakhajorn received allowance from the Medical Food Research Group, Chulalongkorn University.

\section{Authors' contributions}

SS is responsible for conceptualization, data curation, methodology, supervision and editing manuscript. WSis responsible for data curation, analysis and writing and editing manuscript. RS is responsible for data analysisand editing manuscript. All authors have read and approved the manuscript.

\section{Acknowledgements}

The authors would like to express appreciation to all participants for their enthusiasm and collaboration.

\section{References}

1. WHO. Obesity and overweight 2021 [updated 9 June 2021. Available from: https://www.who.int/news-room/fact-sheets/detail/obesity-and-overweight]. (accessed 28 December, 2021)

2. Sakboonyarat B, Pornpongsawad C, Sangkool T, Phanmanas C, Kesonphaet N, Tangthongtawi N, et al. Trends, prevalence and associated factors of obesity among adults in a rural community in Thailand: serial cross-sectional surveys, 2012 and 2018. BMC Public Health. 2020;20:1-9.

3. Huang S, Benchamas G, Huang G. Whole processing and use of rice polishings. Innov Food Sci Emerg Technol. 2020;63:102373.

4. Lai O-M, Jacoby JJ, Leong W-F, Lai W-T. Chapter 2 - Nutritional Studies of Rice Bran Oil. In: Cheong L-Z, Xu X, editors. Rice Bran and Rice Bran Oil: AOCS Press; 2019. p. 19-54.

5. Krishna AGG, Khatoon S, Shiela PM, Sarmandal CV, Indira TN, Mishra A. Effect of refining of crude rice bran oil on the retention of oryzanol in the refined oil. J Am Oil Chem Soc. 2001;78(2):127-31.

6. Godber SJ, Xu Z, Hegsted M, Walker T. Rice bran and rice bran oil. Functional foods development: Fall of Louisiana Agriculture; 2002.

7. Kumari N, Khetarpaul N, Rani V, Rani P. Nutrient composition of full fat and defatted rice bran. Asian J Dairy Food Res. 2018;37(1):77-80.

8. Saisavoey T, Sangtanoo P, Reamtong O, Karnchanatat A. Antioxidant and anti-inflammatory effects of defatted rice bran (Oryza sativa L.) protein hydrolysates on raw 264.7 macrophage cells. J Food Biochem. 2016;40(6):731-40.

9. Wiboonsirikul J, Kimura Y, Kadota M, Morita H, Tsuno T, Adachi S. Properties of extracts from defatted rice bran by its subcritical water treatment. J Agric Food Chem. 2007;55(21):8759-65.

10. Boonla O, Kukongviriyapan U, Pakdeechote P, Kukongviriyapan V, Pannangpetch P, Thawornchinsombut S. Peptides-derived from Thai rice bran improves endothelial function in 2K-1C renovascular hypertensive rats. Nutrients. 2015;7(7):5783-99.

11. Senaphan K, Sangartit W, Pakdeechote P, Kukongviriyapan V, Pannangpetch P, Thawornchinsombut S, et al. Rice bran protein hydrolysates reduce arterial stiffening, vascular remodeling and oxidative stress in rats fed a high-carbohydrate and high-fat diet. Eur J Nutr. 2018;57(1):219-30. 
12. Kahlon TS, Chow FI, Sayre RN, Betschart AA. Cholesterol-Lowering in Hamsters Fed Rice Bran at Various Levels, Defatted Rice Bran and Rice Bran Oil. J Nutr. 1992;122(3):513-9.

13. Newman RK, Betschart AA, Newman CW, Hofer PJ. Effect of full-fat or defatted rice bran on serum cholesterol. Plant Foods Hum Nutr. 1992;42(1):37-43.

14. Association of Official Analytical Chemists (AOAC). Official Methods of Analysis of the Association of Official Analytical Chemists. 16th ed: AOAC International, Gaithersburg; 1999.

15. Pickering TG, Hall JE, Appel LJ, Falkner BE, Graves J, Hill MN, et al. Recommendations for blood pressure measurement in humans and experimental animals: part 1: blood pressure measurement in humans: a statement for professionals from the Subcommittee of Professional and Public Education of the American Heart Association Council on High Blood Pressure Research. Circulation. 2005;111(5):697-716.

16. Amato MC, Giordano C, Galia M, Criscimanna A, Vitabile S, Midiri M, et al. Visceral Adiposity Index (VAI): A Reliable Indicator Of Visceral Fat Function Associated With Cardiometabolic Risk. Diabetes Care. 2010.

17. Woolcott OO, Bergman RN. Relative fat mass (RFM) as a new estimator of whole-body fat percentage - A cross-sectional study in American adult individuals. Sci Rep. 2018;8(1):10980.

18. Padwal MK, Murshid M, Nirmale P, Melinkeri RR. Association of Serum Ferritin Levels with Metabolic Syndrome and Insulin Resistance. J Clin Diagn Res. 2015;9(9):Bc11-3.

19. Katz A, Nambi SS, Mather K, Baron AD, Follmann DA, Sullivan G, et al. Quantitative insulin sensitivity check index: a simple, accurate method for assessing insulin sensitivity in humans. J Clin Endocrinol Metab. 2000;85(7):2402-10.

20. Matthews DR, Hosker JP, Rudenski AS, Naylor BA, Treacher DF, Turner RC. Homeostasis model assessment: insulin resistance and beta-cell function from fasting plasma glucose and insulin concentrations in man. Diabetologia. 1985;28(7):412-9.

21. Committee on DRI. DIETARY REFERENCE INTAKE FOR THAIS 2003: Department of Health, Ministry of Public Health, Thailand 2003. Available from: http://nutrition.anamai.moph.go.th/ewt_news.php?nid=724\&filename=index.

22. Lewis SJ, Heaton KW. Stool form scale as a useful guide to intestinal transit time. Scand J Gastroenterol. 1997;32(9):920-4.

23. Hongu N, Kitts DD, Zawistowski J, Dossett CM, Kopec A, Pope BT, et al. Pigmented rice bran and plant sterol combination reduces serum lipids in overweight and obese adults. J Am Coll Nutr. 2014;33(3):231-8.

24. Wanders AJ, van den Borne JJ, de Graaf C, Hulshof T, Jonathan MC, Kristensen M, et al. Effects of dietary fibre on subjective appetite, energy intake and body weight: a systematic review of randomized controlled trials. Obes Rev. 2011;12(9):724-39.

25. Janika H, Kannan A, Navam SH. Antioxidant and antihypertensive activities of rice bran peptides. Discov Stud J Dale Bump Coll Agric Food Life Sci. 2011;12(1):52-7.

26. Shobako N, Ishikado A, Ogawa Y, Sono Y, Kusakari T, Suwa M, et al. Vasorelaxant and Antihypertensive Effects That Are Dependent on the Endothelial NO System Exhibited by Rice Bran-Derived Tripeptide. J Agric Food Chem. 2019;67(5):1437-42.

27. Weickert MO, Mohlig M, Koebnick C, Holst JJ, Namsolleck P, Ristow M, et al. Impact of cereal fibre on glucose-regulating factors. Diabetologia. 2005;48(11):2343-53.

28. Samra RA, Anderson GH. Insoluble cereal fiber reduces appetite and short-term food intake and glycemic response to food consumed 75 min later by healthy men. Am J Clin Nutr. 2007;86(4):972-9.

29. Kelley DE, Mandarino LJ. Fuel selection in human skeletal muscle in insulin resistance: a reexamination. Diabetes. 2000;49(5):677-83.

30. Weickert MO, Pfeiffer AF. Metabolic effects of dietary fiber consumption and prevention of diabetes. J Nutr. 2008;138(3):439-42.

31. Qureshi AA, Sami SA, Khan FA. Effects of stabilized rice bran, its soluble and fiber fractions on blood glucose levels and serum lipid parameters in humans with diabetes mellitus types I and II. J Nutr Biochem. 2002;13(3):175-87.

32. Cheng HH, Huang HY, Chen YY, Huang CL, Chang CJ, Chen HL, et al. Ameliorative effects of stabilized rice bran on type 2 diabetes patients. Ann Nutr Metab. 2010;56(1):45-51.

33. Vissers MN, Zock PL, Meijer GW, Katan MB. Effect of plant sterols from rice bran oil and triterpene alcohols from sheanut oil on serum lipoprotein concentrations in humans. Am J Clin Nutr. 2000;72(6):1510-5.

34. Kerckhoffs DA, Brouns F, Hornstra G, Mensink RP. Effects on the human serum lipoprotein profile of beta-glucan, soy protein and isoflavones, plant sterols and stanols, garlic and tocotrienols. J Nutr. 2002;132(9):2494-505.

35. Somsuvra BG, Shital JP. Gamma-oryzanol - a multi-purpose steryl ferulate. Curr Nutr Food Sci. 2011;7(1):10-20.

36. Lerma-García MJ, Herrero-Martínez JM, Simó-Alfonso EF, Mendonça CRB, Ramis-Ramos G. Composition, industrial processing and applications of rice bran y-oryzanol. Food Chem. 2009;115(2):389-404.

37. Chen F, Huang S, Huang G. Preparation, activity, and antioxidant mechanism of rice bran polysaccharide. Food Funct. 2021;12(2):834-9.

38. Mudgil D, Barak S. Composition, properties and health benefits of indigestible carbohydrate polymers as dietary fiber: a review. Int J Biol Macromol. 2013;61:1-6.

39. Hebden JM, Blackshaw E, D'Amato M, Perkins AC, Spiller RC. Abnormalities of GI transit in bloated irritable bowel syndrome: effect of bran on transit and symptoms. Am J Gastroenterol. 2002;97(9):2315-20.

40. Jr P, Wu Y. A Review of Physiological Effects of Soluble and Insoluble Dietary Fibers. J Nutr Food Sci. 2016;06.

41. Tomlin J, Read NW. Comparison of the effects on colonic function caused by feeding rice bran and wheat bran. Eur J Clin Nutr. 1988;42(10):857-61.

\section{Figures}

Page $11 / 13$ 


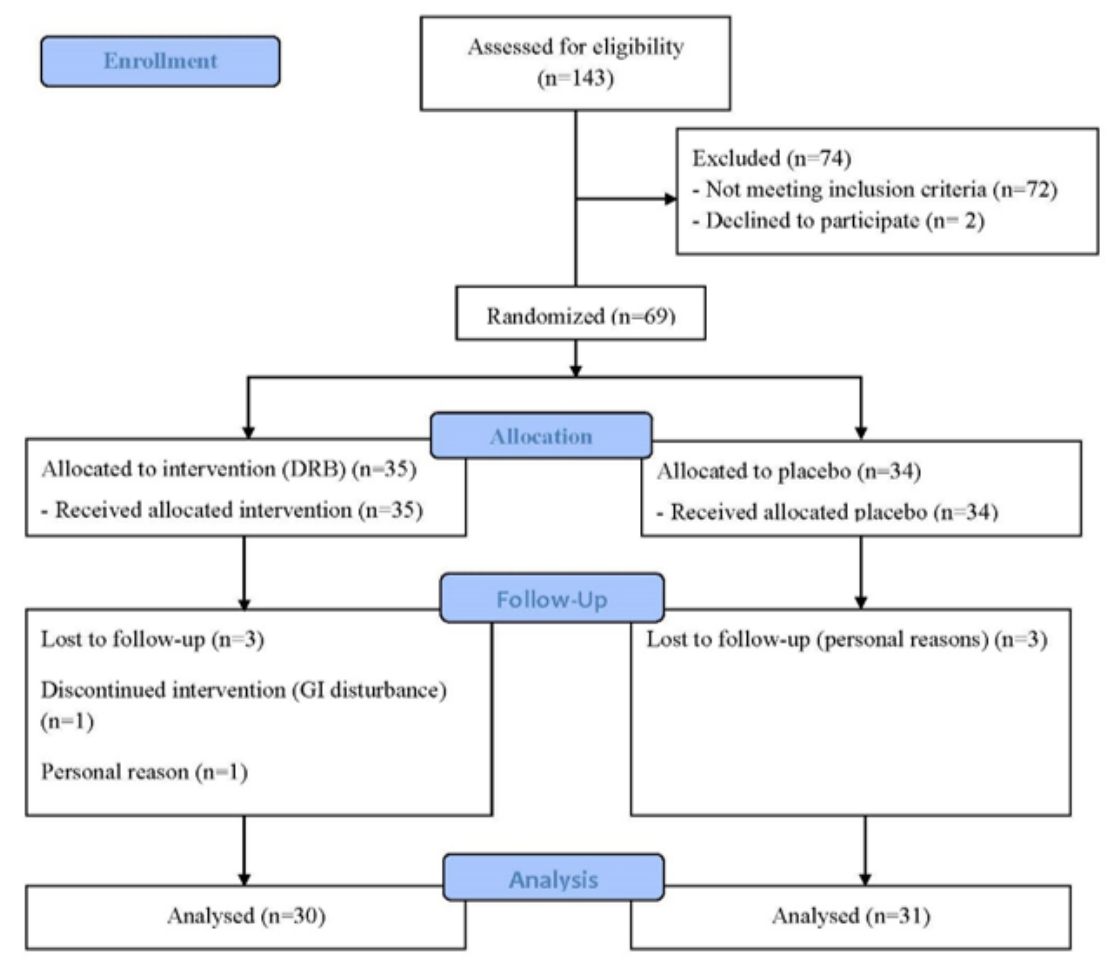

Figure 1

CONSORT flow diagram of the study

(a)

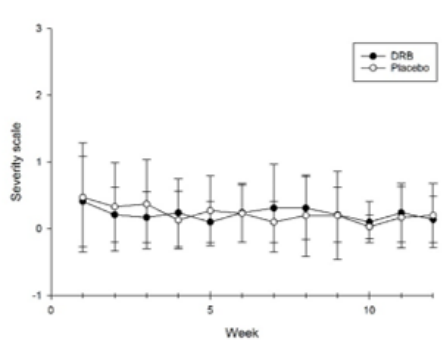

(c)

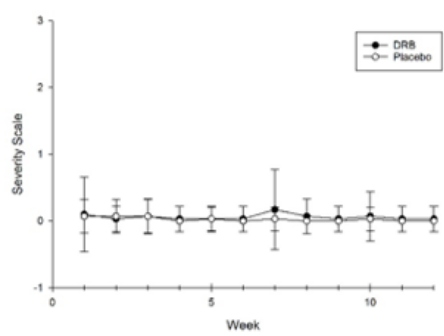

(e)

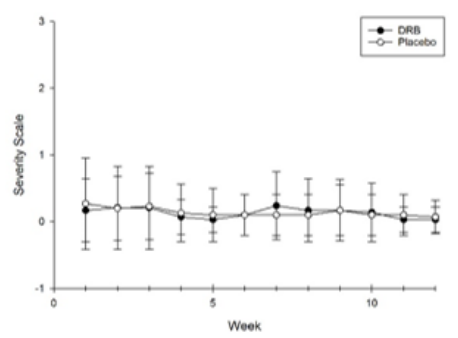

(b) Borborygmi

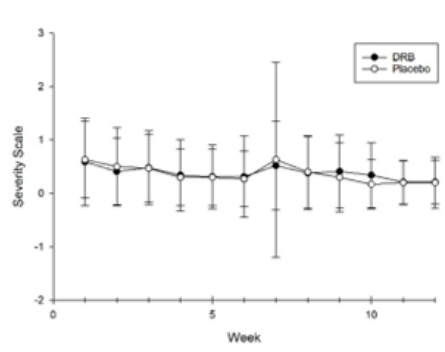

(d)

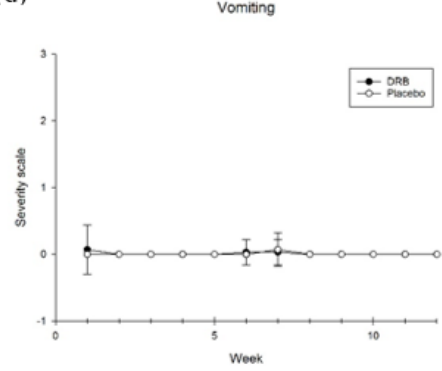

(f)

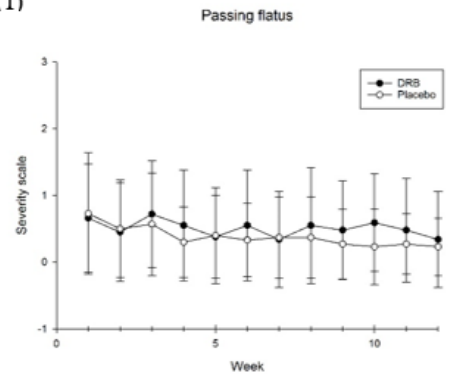


Figure 2

Gastrointestinal symptoms: (a) Flatulence (b) Borborygmi (c) Nausea (d) Vomiting (e) Stomach pain (f) Passing flatus. Mean \pm SD of self-reported gastrointestinal symptoms by participants at each follow-up week. $x$-axis = week of intervention, $y$-axis = intensity of symptoms from 0 (none), 1 (mild), 2 (moderate), and 3 (severe).

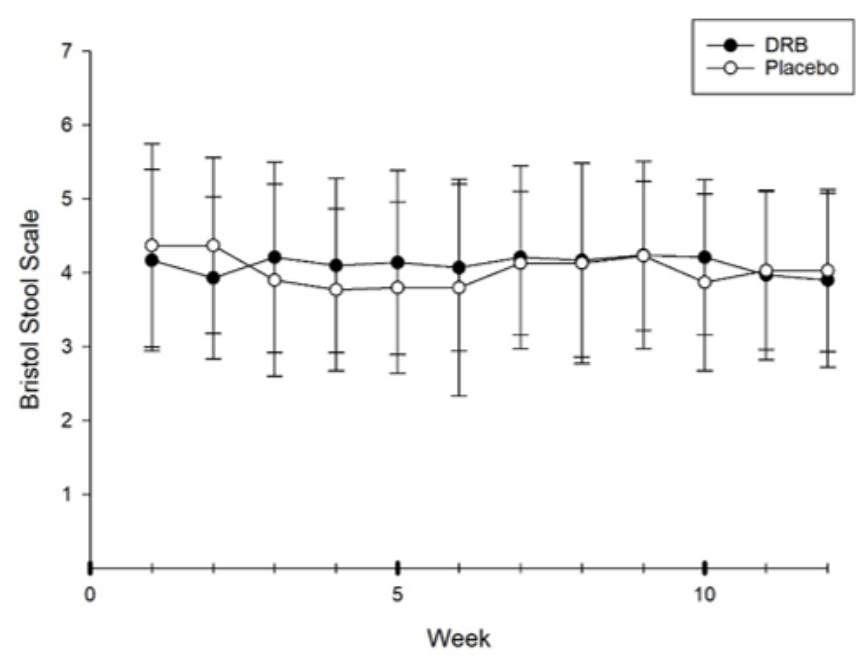

\section{Figure 3}

Bristol stool scale. Mean \pm SD of classification of stool by the Bristol stool scale by participants at each follow-up week. $x$-axis $=$ week of intervention, $y$-axis = type of stool, type 1 = separate hard lumps, type 7 = watery, no solid pieces.

\section{Supplementary Files}

This is a list of supplementary files associated with this preprint. Click to download.

- CONSORT2010Checklist.doc

- GASTROINTESTINALSYMPTOMSEVALUATION.docx 